\title{
MEETINGS
}

Canadian Anaesthetists' Society Western Division Meeting

April 25 and 26, 1980

Four Seasons Hotel Vancouver, B.C.

For Information:

Dr. K. Turnbull

B.C. Anaesthetists' Society

clo Academy of Medicine

1807 West 10th Avenue

Vancouver, B.C.

V6J 2 A9

Maternal ano Perinatal Care 1980 Mount Sinai Hospital

May 16-17, 1980

Toronto, Ontario

For Information:

Dr. E. Hew

Mount Sinai Hospital

600 University Avenue

Toronto, Ontario

M5G 1X5

\section{Canadian Anaesthetists' Society} annual Meeting

June $2 I-25,1980$

Harbour Castle Hilton

Toronto, Ontario

For Information:

Honorary Secretary

178 St. George Street

Toronto, Ontario

M5R 2M7
VIITH WORL D CONGRESS OF ANAESTHESIOLOGY

September $14-21,1980$

Hamburg, Germany

For Information:

Dr. K. Horatz

Hamburg Messe und Congress

Junguiusstrasse

2000 Hamburg 36

Germany

Canadian Anaesthetists' Society

ONTARIO Division

Ontario Medical association

Anaesthetic Section

Annual Fall Meeting

September 26-28, 1980

Airlane Hotel, Thunder Bay, Ontario

For Information:

Dr. J.R. Wilson

328 Catherine Street

Thunder Bay, Ontario

P7E 1K7

Seventeenth Biennial Western Conference ON ANesthesiology Combined Conference and Post-Conference Seminar

$$
\text { May 3-7, } 1981
$$

The Hawaiian Regent Hotel, Waikiki, Hawaii

$$
\text { May 8-9, 1981 }
$$

Intercontinental Hotel, Maui, Hawaii

For Information:

Murrary G. Atnikov, M.D., Secretary-

Treasurer

Biennial Western Conference on

Anesthesiology

Suite 206, 600 Royal Avenue, New

Westminster, B.C.

Canada V3M $1 \mathrm{~J} 3$ 
SECOND INTERNATIONAL Symposium ON

ENDOCRINOLOGY IN ANESTHESIA AND SURGERY

\section{September 1981}

Kyoto, Japan

\section{For Information:}

Dr. T. Oyama

Dept. of Anesthesiology

University of Hirosaki School of Medicine

Hirosaki, Aomori-ken

036, Japan 